\title{
Epigenetics and Systems Physiology of Nutrition: An Overview
}

\author{
Abhay Kumar Pandey , Garima Pandey \\ Department of Physiology, Government Medical College, Banda, Uttar Pradesh, India
}

Copyright $\bigcirc 2017$ by authors, all rights reserved. Authors agree that this article remains permanently open access under the terms of the Creative Commons Attribution License 4.0 International License

\begin{abstract}
Understanding of basic mechanisms mediating epigenetic regulation is essential for knowing cellular differentiation and genomic programming for function. Epigenetics refers to chemical marks on genes and their products, impacting gene expression without any alteration in DNA base sequence. These marks are copied and carried over through cell division and yet, are reversible with appropriate molecular manipulations such as DNA methylation, chromatin rearrangement, microRNA mediated feedback checks etc. Such changes underlie fetal origins of adult chronic non communicable diseases, and project the primacy of nutrition in epigenetic aberrations. Regulation of over 25000 genes through epigenetic processes in health and disease opens vast sphere for physiologic understanding of nutrition and nutrients. Genomic science has systems approach to understanding. In present context that shapes as dynamic emergent nutrition physiology of health and disease. Present article presents a brief overview of the perspectives.
\end{abstract}

Keywords Epigenetics, Nutrigenomics, Molecular Nutrition, Non-communicable Disease

\section{Introduction}

Personalized medicine is based on the principle that each individual is born biologically unique. Genomic understanding is the foundation of personalized medicine. Several genomic methods are currently available to identify susceptibility loci for diseases or phenotypic traits, viz. linkage analysis, candidate gene association studies and genome-wide association studies. By combining these approaches, physicians can predict health risks, determine and quantify the dynamics of disease development and tailor therapeutic protocols to the needs of an individual $(1,2)$. Multicellular organisms have differentiation of cells for task specialization, despite same genetic code, which supports existence of additional information beyond the genetic code. The later appears to regulate genome function. Events and processes effecting heritable changes in gene expression in the dividing cells, which do not involve change in DNA base sequence, are termed epigenetics(3). The epigenetic regulation is critical both for generating diverse cell types and maintaining stability and integrity of genome expression profiles in them. Understanding of basic mechanisms that mediate epigenetic regulation is invaluable to the knowledge of cellular differentiation and genome programming. While the epigenetic changes are heritable and maintained, they are also potentially reversible. Hence, cloning of organism using nuclei of differentiated cells is possible.

\section{Basic Epigenetic Mechanisms}

Mechanisms, such as DNA methylation and chromatin modification have been at focus of studies unraveling molecular basis of epigenetics (4). Both mechanisms act in concert to provide stable and heritable silencing in genome. Epigenetics implies chemical marks on the genome (and associated cellular machinery) that are copied from one cell generation to the next. This may alter gene expression, but no changes are involved in primary DNA sequence. Probability of such marks on genome is described as the epigenome. It comprises of three distinct, but closely interacting mechanisms, including DNA methylation, histone modification and non-coding mi-RNAs(5).The combination of these marks and miRNA, is responsible for regulating gene expression, not only during cellular differentiation in embryonic and fetal development (6), but also through-out life course.

\subsection{Histone Modification}

Dynamics of chromatin functionally links histone modification to epigenetic regulation. Chromatin structure is made up of association of DNA with histone proteins (7). Within the nucleus, DNA is packaged by sophisticated wrapping around the octet of globular proteins, known as histones. The basic repeating unit of chromatin is nucleosome, in which around 147 base pairs of negatively 
charged DNA wrap 1.65 times around a highly positively charged histone protein octamer, consisting of $\mathrm{H} 2 \mathrm{a}, \mathrm{H} 2 \mathrm{~b}, \mathrm{H} 3$ and $\mathrm{H} 4$ histone subunits (8). Additional compaction of nucleosomal DNA occurs by association with linker histone H1, non-histone proteins and looping and folding of chromatin fiber. Such organization of chromatin restricts physical access of nuclear transcription factor to the underlying DNA. Epigenetic marks on the chromatin play central role in regulating the chromatin structure and thereby, accessibility of DNA for transcription.

Post translational modifications viz, acetylation, phosphorylation, methylation, ubiquitination generate marked diversity in the histone/nucleosome structures. These individual histone modifications and/or patterns of modifications described as histone decoration are believed to constitute a histone code (9). The dynamic and reversible modifications such as acetylation and phosphorylation associate with inducible expression of individual genes. Histone code, in conjunction with DNA methylation status and the presence or absence of specific miRNA regulates the expression of associated genes (10). The histones act as signaling platforms that integrate upstream signaling pathways to activation or repression of nuclear transcription (11). The post-translational modifications occur on specific sites in the histones. The proposition that different combinations of histone modifications may result in distinct outcomes in terms of chromatin regulated functions, is called histone code hypothesis (12). The complex super structure overlays the primary genetic information in DNA and, allows sophisticated control of gene expression according to cell and tissue location, time and environment. It governs cell fate decisions both for normal and pathologic development (9) and for ageing (13).

\subsection{DNA Methylation and Other Reactions}

Modifications like methylation are more stable and are involved in the long term maintenance of expression status of regions of the genome. DNA methylation patterns are tissue specific $(14,15)$ DNA methylation is involved in several key physiological processes, including X-chromosome inactivation, imprinting and the silencing of germline specific genes and repetitive elements. These methylation marks are added to the DNA by enzymes of the DNA methyltransferase (DNMT) family, using s-adenosylmethionine (SAM) as methyl donor.

The acetylation of histone is functionally linked to activation of transcription. It is found that during chromosomal rearrangement or transposition the euchromatin genes, juxtaposed to heterochromatin sequences, can become transcriptionally silenced. The extent of silencing defines different phenotypes. The small RNAs are involved in targeting of histone modifying activities to the regions that have to be silenced (16).

\subsection{MicroRNA}

MicroRNA (miRNA) are non-coding RNAs. Some 1046 miRNAs in human genome are documented, encoded on human chromosomes (17). These mRNA regulate transcription of over $30 \%$ of all protein encoding genes, through sequence specific binding to RNA. By controlling expression of about third of human mRNAs, miRNA may influence almost all genetic pathways, by targeting transcription factors, secreted factors, receptors and transporters (18). Variety of dietary factors, ranging from macronutrients (fat, protein, alcohol), to micronutrients (vit E), alter expression of many miRNAs (19). Genomic sequences encoding miRNA are polymorphic (20), a fact that adds further complexity to understand interaction of genome and epigenetics.

\section{Euchromatin and Heterochromatin}

The condense regions of nucleus that do not decondence during interphase, are termed heterochromatin. These are functionally inactive regions of genome. Euchromatin is regions that readily decondense upon exiting mitosis, and actual gene activity takes place in euchromatin. The localization of the histone $\mathrm{H} 2 \mathrm{a}$ variant $\mathrm{H} 2 \mathrm{a} . \mathrm{Z}$, also functions to define boundaries between heterochromatin and euchromatin. H2a. X is a variant from $\mathrm{H} 2 \mathrm{a}$ in human cells, which is rapidly phosphorylated in response to DNA damage. It marks the damaged area, and also attracts the DNA repair complex.

\section{Epigenetic Imprints in Fetal Origin of Adult Diseases}

Epigenetic programming may be the mechanism underlying early life origins of chronic diseases via regulation of gene expression through DNA methylation, histone modification, chromatin remodeling and/or regulatory feedback by way of miRNAs. Epigenetic mechanisms are important because they affect 25000 genes in human genome. Specific forms of genes, called alleles, are recipes for specific protein products which create developmental blueprint. Epigenetic mechanisms can silence genes entirely or cause them to overproduce/overexpress (hypomethylate) or under produce/under express (hypermethylatye) the protein products of genes. The later, influences the downstream biochemical milieu, which underpins all dimensions of complex behavior, including human health.

Different kinds of epigenetic "marks", the chemical additions to genetic sequence are described as "imprint". Imprinted genes do not rely on traditional Mendel's law of inheriting a dominant or recessive trait with equal likelihood of contribution from genes of either parent. Imprinted gene copy comes from any of the parents, frequently, mother. Besides diet, drugs, exercise, epigenetic imprinting may arise from exposure to other individuals akin to their already 
active or redundant genes. Such social imprinting of genes impacts behavior and explains community characters and lifestyle. Epigenetics underlies the plasticity not just of neurons, but also of genes that enable or disable their growth and function.

Growing evidence suggests likelihood of most non communicable diseases (NCDs), being driven by acquired changes in genomic expression over the course of life. Research shows that epigenetic patterns are dynamic in response to both internal and external environmental stimuli throughout life $(21,22)$. Demographic factors as age and gender, environmental exposures (smoking, diet, lifestyle, maternal nurturing, air pollution, microbial infection) and genetic variations can influence epigenetic patterns $(23,24)$. While epigenetic expression patterns can persist over long term, they are potentially reversible. This provides opportunity for optimizing gene expression and health outcomes over the course of life. Folate supplementation in pregnant mother has been found to correct fetal hypomethylation inflicted by environmental endocrine disruptor, bisphenol-A .Impaired glucose metabolism in gestational diabetes increases methylation of fetal leptin gene causing long term risk of diabetes. Epigenetic effects are evident in regulation of key genes involved in adipogenesis, glucose homeostasis, inflammation, insulin signaling etc. Reversible nature of epigenetic alterations promises of therapies that may restore the normal epigenome by activating or silencing disease related genes. Identifying of epigenetic consequences of fetal epigenetic programming can help developing epigenetic biomarkers for early diagnosis of disease, identification of susceptible individuals and development of preventive and curative measures (25).

Whereas DNA variations are permanent, epigenetic changes are in a process of flux and generally accumulate over time. Once a cell DNA pattern is established (whether normal or pathological), the methylated sites are faithfully inherited by daughter cells. New technologies evaluating epigenetic changes, e.g. methylated DNA, miRNA expression, post-translational modifications on histones offer promising biomarkers for understanding and diagnosis of diseases and monitoring therapeutic response. The latter include autoimmune, neurodegenerative, complex multifactorial and rare diseases as well as cancers. If specific epigenetic markers can be established for specific disease, it will be feasible to use blood plasma and perhaps other body fluid samples to analyse epigenetic signatures.

\section{Diet and Epigenetic Modification}

Food is a conditioning environment that shapes the activity of genome and physiology of body. Nutrition in prenatal and early postnatal life impacts adult onset diseases as diabetes, heart diseases. Understanding of how food has become environment in nutritional epigenetics is implied in how food is perceived, manufactured, and its metabolism interpreted. Growing evidence indicates that dietary factors may modify epigenetic marks (26). Gabory et al (27), proposed that environmental, including dietary, factors may induce epigenetic changes via three possible mechanistic pathways: 1) activation/inhibition of chromatin machinery; 2) activation of nuclear receptor by ligands and 3) membrane receptor signaling cascades.

\subsection{Modulation of DNA Methylation}

Dietary and nutritional factors may affect DNA methylation by changing availability of methyl donors and altering the activity of enzymes involved in the process of DNA methylation. One carbon metabolism is central to methylation of biological molecules. Several nutrients affect DNA methylation through this mechanism (28). Nutrient influence on supply of methyl group for one carbon metabolism is most studied. Folate, vit B6, vit B12, choline and methionine are cofactors for enzymes involved in one carbon metabolism. Deficiency or excess of any one may alter the availability of SAM from the methionine cycle. Alteration in SAM availability directly alters DNA methylation. Other dietary nutrients may indirectly affect one carbon metabolism.

Catechol containing dietary polyphenols is excellent substrates for COMT in a SAM consuming reaction. Increased consumption of these food items would decrease SAM availability for DNA methylation (29) Dietary factors as zinc, selenium, genistein and green tea polyphenols, alter DNA methylation by affecting DNA methyltransferase (DNMT) activity (30). Overall, DNA methylation at specific gene loci is positively correlated with supply of dietary methyl donors (31-33). It is negatively correlated to dietary protein intake $(34,35)$. Supplementation of diet with selenium and genistein is associated with tissue specific increase in DNA methylation at selected loci.

Uncertainties prevail about effect of single nutrient (folate) intake indifferent doses and duration of exposure, on alterations in DNA methylation, highlighting need for many systematic studies. Physiological state of subject also impacts changes in DNA methylation. Evidence is building on probably critical time-windows (i.e. during intrauterine life), when nutritional insults would cast particularly large consequences for health $(36,37)$. Altered epigenetic marks lead to changes in gene expression and cellular function. This may be one of the mechanisms, of early life programming of development by dietary and other environmental exposures. Very few studies have included both gene methylation and expression together, in investigating functional consequences of changes in diet/nutrition $(38,39)$. Overall impact of dietary factors on health may be understood by examining effect of diet induced changes on DNA methylation, on gene expression, phenotype and other epigenetic marks.

\subsection{Modulation of Histone Marks}

Dietary factors can alter the post translational 
modification of histones causing alterations in chromatin structure which influences transcription. Histone tails can be modified by diverse processes, viz. methylation, acetylation, phosphorylation, ribosylation, ubiquitination, sumoylation and biotinylation. Diverse multiple scopes exist therefore, for nutrition influence on histone marks. Histone marks may be altered by, 1) altering the abundance and/or efficacy of the enzymes responsible for the modification and 2) altering the availability of the enzyme substrate.

Enzymes carrying out histone modification include histone acetyltransferase (HAT) and histone methyltransferase (HMT). These respectively add acetyl and methyl group to histone. Histone deacetylases (HDAC) and histone demethylases (HDM) remove those groups from histone. Several nutrients may inhibit these enzymes. The short chain fatty acid butyrate produced by colonic fermentation of garlic, soy, cinnamon etc. inhibits HDAC (40). Green tea polyphenols and copper inhibit HAT (41). EGCG (epi-gallocatechine gallate) from green tea inhibits HMT (42). Reduced availability of dietary methyl donors also inhibits HMT activity (43).

DNA methylation and histone modification have joint role in regulation of transcription. Simultaneous evaluation of any dietary/nutrient influences on both the processes is thus necessary. Need for significant cell renewal imposed by chronic stressors may disturb tissue homeostasis and drive chronic disease processes as atherosclerosis, diabetes, cancer etc. (44). Disturbed homeostasis of cellular function may be mediated through epigenetic mechanism. That needs revelation and correction, through probable dietetic means. Epigenetic variations at specific loci associate change in body mass index and obesity (45). Much of current research is focused on very early life. Plasticity of epigenome maybe life-long however, and hence subject to influence of dietary and other exposures. The enormous clinical implications of the later would draw vast research efforts in coming years.

\section{Systems Outlook of Nutrigenomic Physiology}

Systems understanding and application in biosciences implies processes or mechanisms that connect various components of microsystem (molecular, cellular mechanisms) and then integrate them to represent a cohesive co-operative behavior of a system. Any function performed by a system, that is not resultant of a single constituent but of interacting constituents in the system, is termed emergent property (46)Emergent properties can be conceived as group physiology at level of system(organ or organism). Understanding everything is hardly achievable, hence a broad working concept must accept existence of legitimate gray regions of knowledge. This is the useful principle of conservation of uncertainty (47). The notion is that a whole system has a quantity of uncertainty which is always present or constant, but can be shifted around or distributed among components of system and can never be eliminated. Any attempt to increase certainty in some component would automatically increase uncertainty in other. Practical strategies may be designed to cause uncertainty to be distributed in components whose function is less critical for given objective. Present practice of combining information fragments of independent nature, i.e. epidemiological, molecular, transgenic modular or from clinical trials etc. to understand systems function needs advance to incorporate dynamic concept of reality. An example of progress consequent to dynamic pursuit is the understanding that pulsatile insulin release in fundamental to better glycaemic control (48).

- Dietary factors can also interact with other environmental influences in altering DNA methylation patterns and this is high potential research area. Further consideration adding complexity to diet-DNA methylation is tissue specificity. Human genome has over 25000 genes, and diet induced methylation is measured either as overall DNA methylation or methylation of one or few candidate genes. While these studies provide evidence of diet-DNA methylation relationship. They remain just snapshots, incapable of providing glimpse of real picture. Array based investigations may yield data critical in identifying genes or consensus sequences within the genome which are modifiable with individual nutrients. They will also help identifying groups of nutrients that cause similar or opposing epigenetic modifications. Overall impact of dietary factors on health may be determined by characterizing effects of nutritionally mediated changes in DNA methylation on gene expression, phenotype and other epigenetic marks.

\section{REFERENCES}

[1] Pandey AK, Pandey G, Pandey SS and Pandey BL., Human Biology of Diet and Lifestyle Linked Chronic Inflammatory Non-Communicable Disease Epidemic - A Review. Human Biology Review, 2014, 3 (1): 25-42.

[2] Garima Pandey a, Abhay Kumar Pandey Nutrition research perspectives in immune-mediated inflammatory disorders Indian Journal of Rheumatology, 2013, 8: 30-36.

[3] Felsenfeld G. The evolution of epigenetics. Perspect Biol Med. 2014, 57 (1):132-48.

[4] Egger G, Liang G, Aparicio A, Jones PA. Epigenetics in human disease and prospects for epigenetic therapy. Nature. 2004; 429(6990):457-63.

[5] Goldberg AD, Allis CD, Bernstein E. Epigenetics: a landscape takes shape. Cell. 2007 128(4):635-8.

[6] Reik W. Stability and flexibility of epigenetic gene regulation in mammalian development. Nature. 2007; 447(7143):425-32.

[7] Van Holde K Chromatin. NewYork.Springer-Verlag.1998. 
[8] Kornberg RD1, Lorch Y. Twenty-five years of the nucleosome, fundamental particle of the eukaryote chromosome. Cell. 1999; 98(3):285-94.

[9] Jenuwein T, Allis CD. Translating the histone code. Science. 2001; 293(5532):1074-80.

[10] Bernstein BE, Meissner A, Lander ES The mammalian epigenome. Cell. 2007 128(4):669-81.

[11] Cheung P1, Allis CD, Sassone-Corsi P. Signaling to chromatin through histone modifications. Cell. 2000; 103(2):263-71.

[12] Turner BM. Histone acetylation and an epigenetic code. Bioessays. 2000; 22(9):836-45.

[13] Mathers JC1. Nutritional modulation of ageing: genomic and epigenetic approaches. Mech Ageing Dev. 2006; 127(6):584-9.

[14] Ollikainen M1, Smith KR, Joo EJ, Ng HK, Andronikos R, Novakovic B et al DNA methylation analysis of multiple tissues from newborn twins reveals both genetic and intrauterine components to variation in the human neonatal epigenome. Hum Mol Genet. 2010, 19(21):4176-88.

[15] Schneider E, Pliushch G, El Hajj N, Galetzka D, Puhl A, Schorsch $M$ et al Spatial, temporal and interindividual epigenetic variation of functionally important DNA methylation patterns. Nucleic Acids Res. 2010; 38(12):3880-90.

[16] Grewal SI, Moazed D. Heterochromatin and epigenetic control of gene expression. Science. 2003; 301(5634):798-802.

[17] Griffiths-Jones S, Saini HK, van Dongen S, Enright AJ miRBase: tools for microRNA genomics. Nucleic Acids Res. 2008; 36(Database issue):D154-8.

[18] Esquela-Kerscher A1, Slack FJOncomirs - microRNAs with a role in cancer. Nat Rev Cancer. 2006; 6(4):259-69.

[19] Mathers JC1, Méplan C, Hesketh JE. Polymorphisms affecting trace element bioavailability. Int J Vitam Nutr Res. 2010; 80(4-5):314-8.

[20] Ryan BM, Robles AI, Harris CC. Genetic variation in microRNA networks: the implications for cancer research. Nat Rev Cancer. 2010; 10(6):389-402.

[21] Edwards TM1, Myers JP. Environmental exposures and gene regulation in disease etiology Environ Health Perspect. 2007; 115(9):1264-70.

[22] Feinberg AP1. Epigenetics at the epicenter of modern medicine. JAMA. 2008; 299(11):1345-50.

[23] Liu J1, Morgan M, Hutchison K, Calhoun VD A study of the influence of sex on genome wide methylation. PLoS One. 2010 5(4):e10028

[24] Christensen BC1, Houseman EA, Marsit CJ, Zheng S, Wrensch MR, Wiemels JL et al. Aging and environmental exposures alter tissue-specific DNA methylation dependent upon CpG island context. PLoS Genet. 2009 (8):e1000602

[25] Hochberg Z1, Feil R, Constancia M, Fraga M, Junien C, Carel JC et al Child health, developmental plasticity, and epigenetic programming. Endocr Rev. 2011 ;32(2):159-224

[26] Davis CD1, Uthus EO. DNA methylation, cancer susceptibility, and nutrient interactions. Exp Biol Med (Maywood). 2004; 229(10):988-95.
[27] Gabory A, Attig L, Junien C. Sexual dimorphism in environmental epigenetic programming. Mol Cell Endocrinol. 2009 b;304(1-2):8-18

[28] Johnson IT, Belshaw NJ. Environment, diet and CpG island methylation: epigenetic signals in gastrointestinal neoplasia. Food ChemToxicol. 2008 ;46(4):1346-59

[29] Lee WJ, Shim JY, Zhu BT. Mechanisms for the inhibition of DNA methyltransferases by tea catechins and bioflavonoids. MolPharmacol. 2005; 68(4):1018-30.

[30] Fang MZ1, Wang Y, Ai N, Hou Z, Sun Y, Lu H, Welsh W, Yang CS. Tea polyphenol (-)-epigallocatechin-3-gallate inhibits DNA methyltransferase and reactivates methylation-silenced genes in cancer cell lines. Cancer Res. 2003 63(22):7563-70.

[31] Waterland RA1, Jirtle RL. Transposable elements: targets for early nutritional effects on epigenetic gene regulation. See comment in PubMed Commons below Mol Cell Biol. 2003; 23(15):5293-300.

[32] Waterland RA, Dolinoy DC, Lin JR, Smith CA, Shi X, Tahiliani KG. Maternal methyl supplements increase offspring DNA methylation at Axin Fused. Genesis. 2006; 44(9):401-6.

[33] Pogribny IP, Karpf AR, James SR, Melnyk S, Han T, Tryndyak VP Epigenetic alterations in the brains of Fisher 344 rats induced by long-term administration of folate/methyl-deficient diet. Brain Res. 2008; 1237:25-34.

[34] Rees WD1, Hay SM, Brown DS, Antipatis C, Palmer RM. Maternal protein deficiency causes hypermethylation of DNA in the livers of rat fetuses. J Nutr. 2000; 130(7):1821-6.

[35] van Straten EM1, Bloks VW, Huijkman NC, Baller JF, van Meer H, Lütjohann $\mathrm{D}$, et al. The liver X-receptor gene promoter is hypermethylated in a mouse model of prenatal protein restriction. Am J Physiol Regul Integr Comp Physiol. 2010; 298(2):R275-82.

[36] Burdge GC, Lillycrop KA. Bridging the gap between epigenetics research and nutritional public health interventions. Genome Med. 2010; 2(11):80

[37] Junien C Impact of diets and nutrients/drugs on early epigenetic programming. J Inherit Metab Dis. 2006; 29(2-3):359-65.

[38] Bouchard L1, Rabasa-Lhoret R, Faraj M, Lavoie ME, Mill J, Pérusse L, Vohl MC. Differential epigenomic and transcriptomic responses in subcutaneous adipose tissue between low and high responders to caloric restriction. Am J Clin Nutr. 2010; 91(2):309-20.

[39] Thompson RF, Fazzari MJ, Greally JM. Experimental approaches to the study of epigenomic dysregulation in ageing. ExpGerontol. 2010; 45(4):255-68.

[40] Bassett SA, Barnett MP The role of dietary histone deacetylases (HDACs) inhibitors in health and disease. Nutrients. 2014; 6(10):4273-301.

[41] Choi KC1, Lee YH, Jung MG, Kwon SH, Kim MJ, Jun WJ, et al Gallic acid suppresses lipopolysaccharide-induced nuclear factor-kappaB signaling by preventing RelA acetylation in A549 lung cancer cells. Mol Cancer Res. 2009; 7(12):2011-21.

[42] Balasubramanian S, Adhikary G, Eckert RL. The Bmi-1 polycomb protein antagonizes the(-)-epigallocatechin-3-gallat 
e-dependent suppression of skin cancer cell survival. Carcinogenesis. 2010; 31(3):496-503.

[43] Pogribny IP, Tryndyak VP, Muskhelishvili L, Rusyn I, Ross SA. Methyl deficiency, alterations in global histone modifications, and carcinogenesis. J Nutr. 2007; 137(1 Suppl): 216S-222S.

[44] Johnstone SE1, Baylin SB Stress and the epigenetic landscape: a link to the pathobiology of human diseases, Nat Rev Genet. 2010; 11(11):806-12.

[45] Feinberg AP, Irizarry RA, Fradin D, Aryee MJ, Murakami P,
AspelundT, et al. Personalized epigenomic signatures that are stable over time and covary with body mass index. SciTransl Med. 2010; 2(49):49-67.

[46] Naylor S, Cavanagh J. Status of systems biology-does it have a future? Drug Disc Today Biosilica. 2004; 2(5):171-174.

[47] Kitano H. Towards a theory of biological robustness. Mol Syst Biol. 2007; 3:137.

[48] Porksen N Therapy targeting beta cell survival and function in type 2 diabetes mellitus Dabetes Res ClinPract 2006 74(Supl 1):S63-S69. 